\title{
Enabling, predisposing, and reinforcing factors influencing implementation of exclusive breastfeeding among lactating mothers in Limpopo Province, South Africa
}

\begin{abstract}
Background: Breastmilk only is enough for milestone of a child because it contains all necessary nutrients. Because of its benefits World Health Organization recommends that lactating mothers must give their infants only breastmilk for six months and continue up to two years with appropriate complementary food. Despite its benefits breastfeeding is not well practiced in developing countries, South Africa included.
\end{abstract}

Objective: This study aimed to investigate enabling, reinforcing and predisposing factors influencing exclusive breastfeeding interruption among lactating mothers in Limpopo Province.

Methods and materials: This study was cross sectional which employed quantitative measures. Data was collected from 399 lactating mothers during immunization in the selected birthing facilities of Limpopo Province and analysed using SPSS version 26.

Results: The findings indicate that breastfeeding mothers were likely to stop breastfeeding completely when their babies were three months old. The findings were that the majority $72.9 \%$ of the mothers practised mixed feeding and the minority, only $27.1 \%$ practised EBF. It was also found that $38.5 \%$ mothers gave water to the infants, $30.2 \%$ were given soft porridge and $26.1 \%$ formula.

Conclusion: The study revealed that the factors that affect exclusive breastfeeding include: influence from the Parents/in-laws Insufficient breastmilk, religious and cultural beliefs, illness of the mothers and young mothers and going back to work/school.

Keywords: enabling, predisposing and reinforcing factors, exclusive breastfeeding, lactating mothers, implementation
Volume I 3 Issue I - 2022

\author{
Azwinndini Gladys Mudau \\ Department of Public Health, University of Venda, South Africa
}

Correspondence: Azwinndini Gladys Mudau, Master's in Public Health, University of Venda, South Africa, Tel 0794932339 , Email azwinndini.mudau@univen.ac.za

Received: January 03, 2022 | Published: January 10, 2022

\section{Introduction}

Exclusive breastfeeding (EBF) is fundamental for maintaining milestone of a child because it contains all necessary nutrients enough for growth and development. The United Nations and International Children Fund (UNICEF) and World Health Education (WHO) advices lactating mothers to give their infants breastmilk only for six months and continue up to two years and beyond to promote healthy development of children. ${ }^{1}$ Despite EBF benefits and UNICEF/WHO recommendations, South Africa has one of the lowest six-month EBF rates in developing countries.

EBF can act as a remedy to prevent diseases such as upper respiratory tract infection, diarrhoea and ear infections of children under the age of five years and is recommended as the baby's first vaccine that protects children's under-fives diseases and other health problems. Worldwide, only $38 \%$ of babies get the chance of being exclusively breastfed during the first few days of their life and mixed feeding is practiced. ${ }^{2}$ Study conducted in Zimbabwe revealed that many African lactating mothers breastfeed their infants one year and beyond but maintenance of EBF up to six months is not commonly practiced. ${ }^{3}$

In South Africa, very few babies are exclusively breastfed during their first six months of life. Many babies are given solid feeds between two and three months of age and even within a few days of birth. ${ }^{4}$ These practises exposes South Africans to poor health outcomes in both their infant and young child years, as well as adulthood. ${ }^{5}$ Study conducted in Kwazulu-Natal to identify barriers to EBF indicated the influence of the community and elders, grandmothers as the challenge on the implementation of EBF. ${ }^{6}$ A study conducted showed that most women do not breastfeed exclusively because they are afraid not to have enough breastmilk for their infants; mothers perceive that the infant is still hungry and is not being adequately fed with breast milk only. ${ }^{7}$ Other factors which fuelled non-adherence are negative perceptions of breastfeeding among younger women and girls, lack of knowledge, desire for social acceptance and pressure to maintain ideal body shape. ${ }^{8}$ In the North West Province, South Africa, young women choose not to breastfeed because breastfeeding can damage their bodies and breasts. Traditional and cultural factors are strong determinants of breastfeeding in South Africa. In the Northern Cape, cultural beliefs are regarded as another factor influencing EBF, traditional herbs are given to the babies immediately after birth to protect them against witchcraft. ${ }^{6}$ Previous research also shows that lack of knowledge, staff attitudes and perceptions regarding EBF; culture, beliefs, and absence of resources, skills as well as lack of support from the family and high prevalence of HIV contribute to poor performance. ${ }^{9}$ 


\section{Materials and methods}

\section{Study design}

In this study a cross -sectional descriptive design in the quantitative paradigm was employed to investigate factors influencing mothers not adhering to EBF. This design and approach were chosen because they allowed variables to be quantified whilst at the same time allowing data to be collected at one point in time. ${ }^{10,11}$

\section{Study setting}

This study was conducted in Limpopo Province. Out of five districts only two were selected namely Vhembe, and Waterberg. Vhembe is the northernmost district of the province and mostly rural. Most of its 1199856 people speak Venda while the other major languages being spoken in the district are Tsonga, Sepedi and Afrikaans.

Waterberg district is in the western part of the Limpopo Province. Many of its 745758 population speak Sepedi. The traditional health system is very strong in Limpopo Province and communities are still influenced by their cultures.

\section{Population and sampling}

The target population of this study comprised of all lactating mothers of the Limpopo Province. The accessible population comprised all lactating mothers who have infants aged 0-6 months from two selected districts. Multistage sampling was conducted to select the districts, health care facilities and the respondents. Two districts were purposively selected for this study namely, Vhembe and Waterberg districts. The selection was based on the initiation rates disparities. Vhembe had the highest initiation rate of $99 \%$, while Waterberg had the lowest with $78 \%$. Health care facilities from the three selected districts were selected using simple random sampling. The fishbowl technique was used and this involved name of each health facility category (hospital, health centre or clinic) from each selected district being written on a separate slip of paper, placed in a bowl and shuffled. A neutral person was asked to select three health care facilities per district. Per the District Health Information System, the total number of lactating mothers bringing their infants for immunisation was estimated at 2730 monthly. ${ }^{11}$

Based on the target population of 2730, a minimum sample size of $n=349$ was calculated using Slovin's formula $\left[n=N /\left(1+\mathrm{Ne}^{2}\right)\right]$ where $\mathrm{N}$ denotes the total number of lactating mothers and $\mathrm{n}$ is the sample size respectively with $\mathrm{e}=0,05$ as the margin of error. ${ }^{12}$ However, the researcher added 21 respondents to come up with a sample of 400 to cater for non-responses and increase representativeness of the sample.

\section{Instrument and data collection approach}

A questionnaire was employed to collect data. The questionnaire was developed based on the objectives of the study and contained close ended questions written initially in English and given to the language experts to translate it into Tshivenda and Sepedi. A pre-test was carried out by administering the questionnaire to $10 \%$ of respondents who met inclusion criteria in one health care facility of Vhembe district that was not selected in this study (Tshilidzini Hospital) The researcher used the results of the pre-test to identify problems in the design and sequence of questions, corrections were made. The researcher arranged for convenient times to meet with the lactating mothers, preferably after immunisation. Arrangement to use separate rooms for completion of questionnaires were made. The respondents were informed about the study through an information letter and those willing to participate were given a consent form to sign. Those who could not read and write were assisted to complete the questionnaire. It took 30-45 minutes to complete the questionnaire.

\section{Ethical consideration}

Ethical clearance (Project no. SHS/18/PH/25/2108) for the study was obtained from University of Venda Ethics Committee. Permission to conduct the study was sought from the Department of Health, Limpopo Province, Vhembe and Waterberg districts and from the managers of the health care facilities where the study conducted. The names of participants have no bearing on the findings of the study. Therefore, they kept confidential. The researcher made sure that the data from the respondents could not be linked to them, no names were used. Participation depended on the free will of the respondents and they were informed of their right to withdraw from the study at any time, if they wish to. Research conducted in such a manner that respondents were not harmed.

\section{Data analysis}

Data was entered to Microsoft excel and analysed using the Statistical Package of Social Sciences (SPSS) version 26.0. The descriptive statistical method was used to analyse characteristics of respondents. The database used to obtain frequency distribution of the variables and to carry out descriptive analysis. Tables and charts used to summarise the results. Data was coded to reduce its complexity using a code sheet. Correlation analysis was done to test the interdependence of important variables.

\section{Results}

A total of 399 lactating mothers participated in the study with an age range of 18 to 40 years. Table 1 summarises sociodemographic information of the lactating mothers that responded to the questionnaires. The results show that $55 \%$ of the respondents 25 $\%$ were aged 24 to 31 years and the minority $20 \%$ were aged 32 to 40 years. For marital status, the results in show that $51 \%$ were single mothers, $43 \%$ were married and $6 \%$ were divorcees. For employment, the results show that most of the respondents $72.7 \%$ were not formally employed compared to $27.3 \%$ who were employed. The finding is that most of the respondents were not employed. For the qualification, $48.4 \%$ had secondary education and $45.1 \%$ had tertiary education. The finding was that the sample used was highly literate with $93.5 \%$ of the respondents with mainly secondary and tertiary education.

\section{Factors contributing to non-adherence to EBF}

Table 2 shows status of breastfeeding $(n=399)$, the age at which breastfeeding was discontinued $(n=112)$ and the reasons for discontinuing breastfeeding $(\mathrm{n}=104)$.

The results show that $72 \%$ of the respondents continued to breastfeed and $112(28 \%)$ were no longer breastfeeding. Of the 112 respondents who had stopped breastfeeding, $31.3 \%$ did so when the babies were just a month old, $58.9 \%$ stopped within one to three months, $2.7 \%$ within four to five months of the age of the baby, $7.1 \%$ did not indicate when the age of the baby they stopped to breast feed. The findings indicate that lactating mothers were likely to stop breastfeeding when their babies were three months old. Of the 104 respondents indicated the age of babies when they stopped breastfeeding, $57.7 \%$ indicated that they were going back to work, $24.0 \%$ indicated medical condition, $5.8 \%$ going back to school, $1.9 \%$ baby refused the breast, and $10.5 \%$ did not specify the reasons for stopping breastfeeding. The finding was that $28 \%$ of the mothers stopped breastfeeding and of which the majority did so within five months after giving birth, those who stopped breastfeeding, $58.9 \%$ did so when the babies were between one and three months old, the main reason being going back to work, $57.7 \%$. This implies that employment of breastfeeding mothers and medical conditions negatively affected EBF (Table 3). 
Table I Socio-demographic characteristics

\begin{tabular}{|c|c|c|}
\hline Variables & Frequency & Percent (\%) \\
\hline \multicolumn{3}{|c|}{ Age range of respondents in years } \\
\hline 18 to 23 & 221 & 55.4 \\
\hline 24 to 31 & 100 & 25.1 \\
\hline 32 to 40 & 78 & 19.5 \\
\hline Total & 399 & 100 \\
\hline \multicolumn{3}{|l|}{ Marital status } \\
\hline Single & 203 & 50.9 \\
\hline Married & 172 & 43.1 \\
\hline Divorced & 24 & 6 \\
\hline Total & 399 & 100 \\
\hline \multicolumn{3}{|l|}{ Employed } \\
\hline Yes & 109 & 27.3 \\
\hline No & 290 & 72.7 \\
\hline Total & 399 & 100 \\
\hline \multicolumn{3}{|l|}{ Level of education } \\
\hline No formal education & 16 & 4 \\
\hline Primary education & 10 & 2.5 \\
\hline Secondary education & 193 & 48.4 \\
\hline Tertiary education & 180 & 45.1 \\
\hline Total & 399 & 100 \\
\hline
\end{tabular}

Table 2 State breastfeed, age of infants breastfeeding stopped and the reasons for stopping breastfeeding

\begin{tabular}{lll}
\hline & Frequency & $\%$ \\
\hline Still Breastfeeding $\mathbf{n}=\mathbf{3 9 9}$ & & \\
Yes & 287 & 71.9 \\
& & \\
No & 112 & 28.1 \\
Total & 399 & 100
\end{tabular}

Age of baby when stopped breastfeeding $\mathbf{n}=\mathrm{I} / 2$

$\begin{array}{lll}\text { 0-I Month } & 35 & 31.3 \\ \text { I-3 Months } & 66 & 58.9 \\ \text { 4-5 Months } & 3 & 2.7 \\ \text { Abstained } & 8 & 7.1 \\ \text { Total } & 112 & 100\end{array}$

Reasons for stopping breastfeeding $\mathrm{n}=104$

\begin{tabular}{lll} 
Going back to work & 60 & 57.7 \\
Medical condition & 25 & 24 \\
Going back to school & 6 & 5.8 \\
The baby refused the breast & 2 & 1.9 \\
Other, specify & II & 10.6 \\
Total & 104 & 100 \\
\hline
\end{tabular}

Table 3 Liquids and foods given to the baby, the reasons, and the advisor to the mothers

\begin{tabular}{|c|c|c|}
\hline Item & Frequency & Percent \\
\hline $\begin{array}{l}\text { Gave your baby other food or } \\
\text { liquid like juice apart from breast }\end{array}$ & Milk (n =399) 291 & \\
\hline Yes (Mixed feeding) & & 72.9 \\
\hline No (EBF) & 108 & 27.1 \\
\hline Total & 399 & 100 \\
\hline \multicolumn{3}{|l|}{$\begin{array}{l}\text { Liquid given to baby } n=29 \text { I } \\
\text { Water }\end{array}$} \\
\hline & 112 & 38.5 \\
\hline Soft porridge & 88 & 30.2 \\
\hline Formula & 76 & 26.1 \\
\hline Other & 15 & 5.2 \\
\hline Total & 291 & 100 \\
\hline $\begin{array}{l}\text { Reasons for introducing other } \\
\text { food or liquid other than breast }\end{array}$ & Ik $(n=291) 95$ & \\
\hline $\begin{array}{l}\text { mi Breast milk was not enough for } \\
\text { the baby }\end{array}$ & & 32.6 \\
\hline Religious and cultural beliefs & 64 & 21.9 \\
\hline Milk was not coming out & 22 & 7.6 \\
\hline Illness of the baby & 40 & 13.7 \\
\hline Illness of the mother & 58 & 19.9 \\
\hline Baby was crying & 12 & 4.1 \\
\hline Total & 291 & 100 \\
\hline $\begin{array}{l}\text { Advisor to give your child other } \\
\text { food/fluid other than breast milk }\end{array}$ & $(n=29 I) \mid 42$ & \\
\hline Parents/ Parents in law & & 48.7 \\
\hline Own decision & 63 & 21.6 \\
\hline Health care workers & 39 & 13.4 \\
\hline Baby' father & 34 & 11.6 \\
\hline Relatives & 8 & 2.7 \\
\hline Other & 5 & 1.7 \\
\hline Total & 291 & 100 \\
\hline
\end{tabular}

The findings were that $72.9 \%$ of the mothers practised mixed feeding and the minority, $27.1 \%$ practised EBF. It was also found that $38.5 \%$ mothers gave water to the infants, $30.2 \%$ were given soft porridge and $26.1 \%$ formula, and $5.2 \%$ did not specify the food given. There were no clear major reasons for mixed feeding though insufficient breast milk for the baby was mentioned, with $32.6 \%$, respondents and religious and cultural beliefs by $21.9 \%$ of respondents as well as poor lactation $7.6 \%$ appeared to be influencing alternative feeding. The finding was that all the reasons were valid, but the most compelling one was not enough breastmilk and religious and cultural beliefs, illness of the mother.

It was also found that $48.7 \%$ of the respondents were advised by their parents or parents in-law to practice mixed feeding while $21.6 \%$ respondents decided to act on their own, health care workers $13.4 \%$ and fathers of the babies $11.6 \%$ were found to be contributing far less than what was expected. Poor lactation by mothers and their traditional beliefs held by mothers and parents/parents-in-law were the main factors affecting the implementation of EBF in Limpopo 
Province. Unprofessional advice was also used to make crucial decision on mixed feeding as health care workers advised only $13.4 \%$ of the mothers.

Figure 1 shows results on who influences mothers on adopting a certain type of feeding. The results show that mothers in-law/parents had the most influence as indicated by $31.8 \%$ respondents, $30.8 \%$ respondents indicated that they made own decisions on which type feeding style to use, health workers influenced $22.6 \%$, while $13.8 \%$ respondents were influenced by their husbands/spouses.

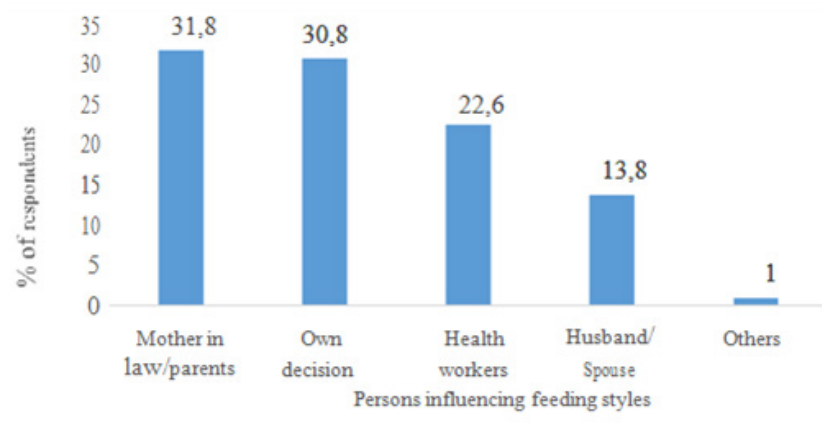

Figure I Persons who influence respondents on feeding styles.

\section{Relationships between variables of the study}

Chi-square tests to check the presence and strength of association and the statistical significance was defined at a probability level of 5\% and conducted for selected variable. Only results where there was a significance level of $\mathrm{p}<0.05$ were presented and reported on.

Pearson's rho (r) correlation coefficient was used in this study. Correlation tests show relationships between factors influencing EBF and selected variables which influenced EBF among lactating mothers. Correlation analysis results show a relationship between continuing feeding and the demographic characteristics of the mothers namely age of infant, employment as well as age of infant and mode of delivery.

Infant's age \& still breastfeeding: Continuing breastfeeding was significantly negatively correlated with the age of the infancy at $\mathrm{p}<0.01, \mathrm{r}=-0.323$. This implied that breastfeeding was likely to be stopped when the age of the child increased. Age of the child was a factor that influenced EBF.

Employment \& Q still breastfeeding: There was a significant relationship between the tendency of mothers to continue breastfeeding and employment at $\mathrm{p}<0.01, \mathrm{r}=-0.381$, showing that employed mothers were more likely to stop breastfeeding compared to unemployed mothers. Employment of breastfeeding mothers was likely to disrupt the implementation of EBF.

Qualification \& still breastfeeding: A negative correlation existed between the qualification of mothers and continuing breastfeeding, with $\mathrm{r}-0.114$ at $\mathrm{p}<0.05$. Mothers with higher educational level were more likely to stop breastfeeding earlier than those with lower qualifications. The implementation of EBF in Limpopo Province is negatively affected by the educational level of the mothers with those higher qualifications stopping breastfeeding to return to work within a two- or three-months period because maternity leave is only four months in South Africa.

Sex infant \& still breastfeeding: The continuation of breastfeeding beyond six months was also related to the gender of the child at $\mathrm{r}=0.201, \mathrm{p}<0.010$, with mother preferring to continue breastfeeding boys to girls. This preference influenced EBF by promoting it if most the babies were boys and disrupts it if most the babies were girls.

Place delivery \& still breastfeeding: A weak negative correlation existed between the place of birth and continuing breastfeeding, $r=-$ $0.121, \mathrm{p}<0.05$, with mothers delivering at home more likely to stop breastfeeding before a period of six months than those who gave birth in hospitals. The place of delivery influences the continuation of breastfeeding to a certain extent.

Correlation analysis was also performed on demographic variables of mothers and stopping of breastfeeding. The values for $r$ and $p$ show relationships that were significant at $\mathrm{p}<0.05$ and $\mathrm{p}<0.01$.

Age \& stopped breastfeeding: The results show a moderate relationship $\mathrm{r}=0.220$ at $\mathrm{p}<0.05$ that stopping breastfeeding was directly linked to the age of the mothers. Elder mothers were likely to prolong breastfeeding compared to younger mothers. This confirms that age of mothers was an influential factor in the implementation of breastfeeding among mothers.

Marital status \& stopped breastfeeding: With $r=0.301$ at $p<0.05$, a significant positive relationship exists between marital status of mothers and stopping breastfeeding, with single and divorced mothers more likely to stop breastfeeding within a short period compared to married mothers. Divorced and single mothers could have been compelled to do this by other circumstances such as returning to work immediately to sustain the families or lack of pressure to continue breastfeeding usually by husbands or partners. This implies that marital status is an influential factor to the implementation EBF in the province. Married mothers were more likely to get support to keep on breastfeeding from their spouses who will go and work to raise basic needs for the family unlike with single and divorcees.

Number children \& stopped breastfeeding: There was a significant strong and positive relationship between the stopping breastfeeding and number of children, $\mathrm{r}=0.46$, a $\mathrm{p}<0.01$. Mothers with many children were more likely to stop breastfeeding within a short period after birth. Number of children a mother had was an influential factor in the implementation of EBF in the district.

Working \& stopped breastfeeding: A strong and positive significant relation existed between employment of mothers and stopping breastfeeding, $r=0.603, p<0.01$ implying that employed mother were more likely to stop breastfeeding after delivery. Employment of mothers was one of the major factors influencing the implementation of EBF in the district. Employment of breastfeeding mothers led to the disruption of the EBF programme in the district. Working mothers stay away from babies for long periods and would also afford to buy supplement foods instead of using express milk.

Qualification \& stopped breastfeeding: There also a strong and positive relationship between the qualification of mothers and stopping breastfeeding, $\mathrm{r}=0.545$ at $\mathrm{p}<0.01$, implying that the higher educational qualification the more likely the mother would stop breastfeeding. In many communities, women with high qualifications are employed and usually are expected to return to work immediately especially divorces and single mothers. High educational level of breastfeeding mothers can be construed as a factor disrupting EBF as it leads to their employment which separates them from babies for long periods of times.

Infant's age \& stopped breastfeeding: There is a strong relationship of the infancy significantly related to stopping breastfeeding, $\mathrm{r}=0.608$, $\mathrm{p}<0.01$. As the age of the infants increased, breastfeeding was more likely to be discontinued and replaced with solid foods. 
Sex of an infant $\&$ stopped breastfeeding: With $\mathrm{r}=-0.416$, at $\mathrm{p}<0.01$, a negative significant relationship exists between gender of the child and stopping breastfeeding. This implies that female infants were more likely to be stopped breastfeeding that their male counterparts.

\section{Discussion}

Factors that potentially facilitate the implementation of EBF are: predisposing factors which include attitudes, beliefs, personal preferences, skills, self-efficacy towards the desired behaviour change; Enabling factors are skills or physical factors such as availability and accessibility of resources, or services that facilitate achievement of motivation to change behaviour. The opinions and support of those surrounding somebody every day can influence the behaviour and the attitude of the individual. Friend and family can make breastfeeding easier by providing emotional support and relevant personal experiences or make it more difficult by expressing displeasure or behaving in ways that counter breastfeeding success; Reinforcing factors include factors that reward or encourage the positive behaviour change, including social support, economic reward and changing the social norm. ${ }^{13}$

The findings were that majority of the mothers practised mixed feeding and the minority practised EBF. Cultural and religious beliefs have been identified as the factors influencing EBF in Limpopo Province. Infants are given anointing water and herbal soft porridge for protection. This influence is probably influenced by parents and parent's in-law and is a common practice among the communities in the province. The study conducted in Ghana also revealed that the low practice of EBF can be attributed to religious, cultural and traditional beliefs. ${ }^{14}$ This is also contrary to a study conducted in Ethiopia where mothers squeezed the colostrum and poured it away due to the Ethiopian cultural beliefs that colostrum can cause abdominal pains in their infants. ${ }^{8}$ Parents, parents' in-law, Friends and relatives usually encourage lactating mothers to give water and other traditional herbs to infants as a perceived way of quenching their thirst or as a sign of welcoming them into the world. ${ }^{14,15}$ Poor lactation by mothers and traditional beliefs held by mothers and parents/parents-in-law were major factors affecting the implementation of EBF in Limpopo Province. This finding was the same with the study that was conducted in China were young mothers were less likely to practice EBF due to general traditional practice of mix feeding especially in rural areas. ${ }^{4}$

Furthermore, the study found employment as a contributory factor to implement EBF because maternity leave is only four months in South Africa. Due to a short maternity leave employed lactating mothers tend to introducing solids food earlier because they should go back to work, this notion supported by the study which reported low prevalence among educated Nigerian mothers may be attributed to current economic challenges in Nigerian, where mothers may be forced to return to full time work causing a shorter duration of breastfeeding. ${ }^{16}$ In the US, workplace policy surrounding breastfeeding and parental leave often do not reflect these benefits. In addition, women are often unable to risk the loss of their jobs or loss of income due to EBF adherence, so formula feeding is the best option for them. ${ }^{17}$

Age of the mother is considered the major factor influencing EBF. In this study, EBF rate is higher to older mothers than in younger mothers this is in line with previous study that was conducted in Zimbabwe found that EBF rates were higher among mothers aged 35-39 compared to those less than 20 years old. Higher rates were also found among 25-34 and 35-36 years old compared to younger mothers. ${ }^{18}$ The results show a moderate relationship $\mathrm{r}=0.220$ at $\mathrm{p}<0.05$ that stopping breastfeeding was directly linked to the age of the mothers. Elder mothers were likely to prolong breastfeeding compared to younger mothers. This confirms that age of mothers was an influential factor in the implementation of breastfeeding among mothers. Similarly, Study which was conducted in Zimbabwe which concluded that experience is the best teacher since it increased likelihood to EBF, adolescence mothers were less likely to practice EBF in Zimbabwe. ${ }^{4}$ This finding was also consistent with Chinese study where mothers of the age group 14-24years were less likely to practice EBF. ${ }^{19}$

Perceptions of the mothers and family members that breastmilk only is not enough. A study conducted in South Africa showed that most women do not breastfeed exclusively because they are afraid not to have enough breastmilk for their infants; mothers perceive that the infant is still hungry and is not being adequately fed with breast milk only. ${ }^{7}$

Medical condition of the mother was found to be responsible for decrease in the duration and exclusivity of breastfeeding. This is confirmed in the study conducted Uganda which found a positive association between decreased duration of breastfeeding and positive status of the mother due to fear of mothers transmitting the virus to their infants. ${ }^{20}$ Fear of transmission of $\mathrm{HIV}^{+}$mothers to their infants was alluded as a major hindrance to EBF. In the study done on perception of the role of maternal nutrition in $\mathrm{HIV}^{+}$lactating breastfeeding women, it was reported that most women perceived $\mathrm{EBF}$ as a factor that may increase the progression of $\mathrm{HIV} .{ }^{20} \mathrm{~A}$ maternal HIV positive status was found to be significantly associated with the practice of EBF in Zimbabwe. ${ }^{4}$

With $r=0.301$ at $p<0.05$, a significant positive relationship exists between marital status of mothers and stopping breastfeeding, with single and divorced mothers more likely to stop breastfeeding within a short period compared to married mothers. Divorced and single mothers could have been compelled to do this by other circumstances such as returning to work immediately to sustain the families or lack of pressure to continue breastfeeding usually by husbands or partners. This implies that marital status is an influential factor to the implementation EBF in the district. Married mothers were more likely to get support to keep on breastfeeding from their spouses who will go and work to raise basic needs for the family unlike with single and divorcees. Dependent syndrome often limits the mother sense of selfdetermination. Mothers who are dependent to their husbands often do not have power, they adhere to their family's advices on infant feedings because their in-laws or partners impose what to be given to the infant. ${ }^{21}$

There is also a strong and positive relationship between the qualification of mothers and stopping breastfeeding, $r=0.545$ at $\mathrm{p}<0.01$, implying that the higher educational qualification the more likely the mother would stop breastfeeding. In many communities, women with high qualifications are employed and usually are expected to return to work immediately especially divorces and single mothers. High educational level of breastfeeding mothers can be construed as a factor disrupting EBF as it leads to their employment which separates them from babies for long periods of times.

This study revealed that as the age of the infants increased, breastfeeding was more likely to be discontinued and replaced with solid foods. A recent study Nigeria also supported the finding that age and sex of the baby predicted improved EBF. ${ }^{16}$ This implies that the continuation of breastfeeding beyond six months was also related to the gender of the child with $\mathrm{r}=-0.416$, at $\mathrm{p}<0.01$, a negative significant relationship exists between gender of the child and stopping breastfeeding. This implies that female infants were more likely to be stopped breastfeeding that their male counterparts. 
The place of delivery influences the continuation of breastfeeding to a certain extent, with mothers delivering at home more likely to stop breastfeeding before a period of six months than those who gave birth in hospitals. The EBF implementation of mothers who delivered at birthing facilities was high compared with mothers who delivered at home. Reasons could be the impact of information on EBF that they received from the healthcare workers. However, this finding differed with findings from $\mathrm{Canada}^{22}$ where mothers delivered at home were more likely to continue with EBF for six months.

Mothers with many children were more likely to stop breastfeeding within a short period after birth. Number of children a mother had was an influential factor in the implementation of EBF in the province. This notion contradicted with the previous study where they concluded that multi-parity was a major factor contributing to EBF implementation. ${ }^{23}$ The two studies conducted in Brazil also concluded that being a novice lactating mother was also found out to be a factor to EBF. ${ }^{8}$ Mother's confidence with previous pregnancies could be due to previous positive experiences in breastfeeding as well as previous negative outcome of early introduction of solids. ${ }^{24}$

\section{Conclusion}

Factors such as age of the mother, employment, mothers' illness, cultural and religious beliefs, influence from parents/in-laws and economic status was found to influence the practice of EBF in South Africa. Efforts to empower women and promotion of EBF practices are recommended.

\section{Acknowledgments}

We would like to thank lactating mothers who participated in this study and health care facilities for allowing us accessibility to collect data.

\section{Funding}

This research project was funded by Research Publication Committee (RPC), University of Venda.

\section{Conflicts of interest}

The author declares that they have no competing interests.

\section{References}

1. WHO, UNICEF. Baby Friendly Hospital initiative; Revised, updated and expanded for integrated care WHO. Geneva. 2009.

2. United Nations Children Fund (UNICEF), World Health Organization (WHO). The baby friendly hospital initiative in the United States.

3. WHO. Division of child health and development; evidence for the ten steps to successful breastfeeding. Geneva: World Health Organization; 2016.

4. Mundagowa PT, Chandambuka EM, Chimberengwa PT, et al Determinants of exclusive breastfeeding among mothers of infants aged 6 to 12 months in Gwanda District, Zimbabwe. Int Breastfeed J. 2019;14:30.

5. Du Plessis L, Peer N, Honikman S, et al. Breastfeeding in South Africa: are we making progress? SAHR. 2016.

6. Siziba LP, Hanekom SM, Wentzel-Viljoen E. Low rates of exclusive breastfeeding are still evident in four South African provinces. South African Journal of Clinical Nutrition. 2015;28(4):170-179.
7. Van der Merwe M, Du Plessis LM, Jooste H. Comparison of infantfeeding practices in two health sub-districts with different baby-friendly status in Mpumalanga province. S Afr J Clin Nutr. 2015;28(3):121-127.

8. Liben ML, Gemechu YB, Adugnew M, et a. Factors associated with exclusive breastfeeding among mothers in Dubti town, afar regional state, northeast Ethopia: a community based cross-sectional study. International Breastfeeding Journal. 2016;11(4).

9. West C, Renz H, Jenmalm MC. The microbiota and inflammatory noncommunicable diseases: Associations and potentials for gut microbiota therapies, Journal of Allergy and Clinical Immunology. 2015;135(1):3-13.

10. Brink H, Vander Walt C, Van Ransburg G. Fundamentals of research Methodology for health care professionals. 3rd edn. Juta. Cape Town; 2012.

11. Groove SK, Burns N, Gray JR. The practice of nursing research. Appraisal, synthesis and generation of evidence 7th edn. Elsevier; 2013.

12. Guilford JP, Frucher B. Fundamental statistics in psychology and education. New York: MC Graw-Hill; 1973.

13. Victor R, Baines Sk, Agho KE, et al. Determinants of Breastfeeding indicators among children less than 24 months of age in Tanzania: a secondary analysis of the 2010 Tanzania demographic and health survey. BMJ. 2013;(3):1-8.

14. Otoo GE, Lartey AA, Perez-Escamilla R. Perceived incentives and barriers to exclusive breastfeeding among peri-urban Ghanaian women. J Hum Lact. 2009;25(1):34-41.

15. Iddrisu S. Exclusive breastfeeding and family influences in rural Ghana: a qualitative study. Malmö, Sweden: Malmö University; 2013.

16. Agho KE, Dibley MJ, Odiase JI, et al. Determinants of exclusive breastfeeding in Nigeria; BMC pregnancy and child birth. 2011.

17. Smith JB, Nii AC, Judith AF, et al. The impact of traditional birth attendant training on delivery complications in Ghana. Health Policy Plan. 2000;15(3):326-331.

18. Munjoma TP. Socio-demographic factors associated with exclusive breastfeeding among mothers with children less than six months of age in Zimbabwe, Wits school of Public Health, South Africa; 2015.

19. Dashti M, Scott JA, Edwards CA, et al. Predictors of breastfeeding duration among women in Kuwait: Results of a prospective cohort study. Nutrients. 2014;6(2):711-728.

20. Bodo B. Strategies to promote exclusive breastfeeding in the post conflict rural Northern Uganda. University of Leeds; 2016.

21. Saka FJ. Factors influencing exclusive breastfeeding among HIV positive mothers at Ilala Municipality. Dar-Es Salaam, Master of Public Health Dissertation, Muhimbili University of Health and Allied Sciences; 2012.

22. Senarath U, Dibley MJ, Agho KE. Breastfeeding practices and associated factors among children under 24 months of age in Timor-Leste. Eur J Clin Nutr. 2007;61(3):387-397.

23. Victora CG, Bahl R, Barros AJD, et al. Breastfeeding in the $21 \mathrm{st}$ century: epidemiology, mechanisms, and lifelong effect. Lancet. 2016;387(10017):475-490.

24. Asemahagn MA. Determinants of exclusive breastfeeding practices among mothers in Azezo district, northwest Ethiopia. Int Breastfeed J. 2016;11:22 